\title{
С.В. Бакушев
}

\section{ДИФФЕРЕНЦИАЛЬНЫЕ УРАВНЕНИЯ РАВНОВЕСИЯ СПЛОШНОЙ СРЕДЫ ДЛЯ ПЛОСКОЙ ДЕФОРМАЦИИ В ЦИЛИНДРИЧЕСКИХ КООРДИНАТАХ ПРИ БИЛИНЕЙНОЙ АППРОКСИМАЦИИ ЗАМЫКАЮЩИХ УРАВНЕНИЙ}

\begin{abstract}
Рассматривается построение дифференциальных уравнений равновесия в перемещениях для плоского деформирования сплошных сред при билинейной аппроксимации замыкающих уравнений без учёта геометрической нелинейности в цилиндрической системе координат. Построение билинейных физических зависимостей основано на вычислении секущих модулей объёмного и сдвигового деформирования. При этом на первом участке диаграмм секущий модуль и объёмного и сдвигового деформирования постоянен, в то время как на втором участке диаграмм секущий модуль объёмного деформирования является функцией объёмной деформации, а секущий модуль сдвига является функцией интенсивности деформаций сдвига.
\end{abstract}

Ключевые слова: сплошная среда, плоская деформация, иилиндрические координаты, дифференциальные уравнения равновесия, билинейные замыкаюшие уравнения, геометрически линейная модель.

Вопросы построения замыкающих уравнений для сплошных нелинейно упругих сред, находящихся в условиях сложного напряжённого и деформированного состояния, позволяющие адекватно описать эволюцию их напряжённо-деформированного состояния, являются ключевыми $[1,2]$. Фактические зависимости между объёмными деформациями и объёмным напряжениями, а также между сдвиговыми деформациями и сдвиговыми напряжениями являются, как правило, нелинейными и трудно поддающимися аналитическому описанию. Вместе с тем, даже в случае нелинейного аналитического описания замыкающих уравнений, решение конкретных задач методами теории упругости является чрезвычайно сложным процессом, обусловленным физической нелинейностью.

Для исключения эффектов физической нелинейности предлагается диаграммы объёмного $\sigma=\sigma(\varepsilon)$ и сдвигового $T=T(\Gamma)$ деформирования при сложном напряжённо-деформированном состоянии аппроксимировать билинейными функциями. При этом и на первом и на втором участках билинейных диаграмм объёмного и сдвигового деформирования можно будет использовать уравнения линейной (или геометрически нелинейной) теории упругости. Это с одной стороны. А с другой - разрешающие дифференциальные уравнения и системы дифференциальных уравнений, в силу линейных зависимостей между напряжениями и деформациями, будут иметь относительно простой вид.

Вопросам аппроксимации диаграмм работы материалов уделяется пристальное внимание. Так, в работе [3] рассмотрены практические вопросы аппроксимации опытных диаграмм работы неупругих материалов степенными и дробнолинейными функциями. Дана методика определения геометрического и физического смысла эмпирических коэффициентов, входящих в аппроксимирующие 
формулы. В статье [4] показано, что результаты расчётов цилиндрической оболочки с сыпучим заполнителем при поперечном изгибе в геометрически и физически нелинейной постановке существенно отличаются от экспериментальных данных, если применяется билинейная аппроксимация диаграммы деформирования без учёта критических значений напряжений и деформаций. Отмечено, что точность решения во многом определяется величиной касательного модуля упрочнения материала. В работе [5], на основе положения о кинематической и статической определимости, описана методика определения диаграмм нелинейного деформирования разносопротивляющихся материалов при неоднородном напряжённо-деформированном состоянии, а также иллюстрируются основные приёмы (сплайн-аппроксимации) для достижения приемлемой точности результирующих функций. В работе [6] рассматриваются и анализируются физические законы связи напряжений и деформаций современной теории упругопластического деформирования, а также постулаты макроскопической определённости и изотропии начально-изотропных сплошных сред. Обсуждается вопрос о возможности применения постулата изотропии к оценке влияния параметров вида напряжённодеформированного состояния, возникающего из-за деформационной анизотропии при изменении внутренней структуры материалов. Также обсуждается вопрос о правомерности представления симметричных тензоров второго ранга напряжений и деформаций в виде векторов координатного линейного евклидова шестимерного пространства. Предложен соответствующий принцип тождественности тензоров и векторов. В статье [7] для случая одноосного деформирования реологически сложных сред с учётом разносопротивляемости деформациям растяжения и сжатия приводится общая схема построения математических моделей, на базе которых строятся модели для пространственного напряжённо-деформированного состояния и обсуждаются вопросы идентификации входящих в них механических постоянных.

В настоящее время в связи с усложнением форм строительных конструкций, развитием авиастроения, кораблестроения, ракетостроения и так далее роль теории упругости как в линейной, так и в нелинейной постановке, резко изменилась. Теперь она составляет основу для построения практических методов расчёта деформируемых тел и систем тел разнообразной формы. При этом в современных прочностных и деформационных расчётах учитываются не только сложность формы тела и разнообразие воздействий (силовое, температурное и т.п.), но и специфика физических свойств материалов, из которых изготовлены тела.

Значительное внимание уделяется расчёту деформируемых тел с учётом геометрической и физической нелинейности. Так, в работе [8] на основе соотношений нелинейной теории упругости, в предположении простого активного нагружения, получено решение физически и геометрически нелинейной задачи о больших деформациях полой сферы, выполненной из несжимаемого материала. Полученные результаты свидетельствуют о существенном влиянии физической нелинейности материала сферы на функциональную зависимость перемещение - давление. Автором [9] разработана теория расчёта стержневых конструкций с учётом всех видов нелинейности. Изогнутая ось стержня аппроксимируется пространственной кривой. Получены системы разрешающих дифференциальных уравнений. В статье [10] приведен динамический критерий потери устойчивости прямоугольных в плане сферических оболочек при действии переменной во времени поперечной нагрузки с учётом двух типов нелинейности - геометрической и физической. Геометрическая нелинейность учитывается на основе соотношений 
Т. Кармана. Физическая нелинейность описывается деформационной теорией пластичности А.А. Ильюшина. В статье [11] представлен вычислительный алгоритм, одновременно учитывающий физическую и геометрическую нелинейность деформирующейся среды, предназначенный для изучения статического упругопластического состояния вблизи транспортных подземных выработок произвольной формы. В основу алгоритма положена вариационная формулировка нелинейного конечно-элементного анализа. В работе [12] на основе гипотезы Эйлера Бернулли построена теория нелинейной динамики неоднородной однослойной балки с учётом физической геометрической (в смысле Т. фон Кармана) нелинейности. Балка находится под действием знакопеременной нагрузки, распределённой равномерно по всей её поверхности. В статье [13] рассматриваются пологие оболочки на прямоугольном плане с учётом геометрической и физической нелинейности, а также неоднородности свойств материала по толщине оболочки. Авторами [14] рассмотрены процессы деформирования шарнирно закреплённых по торцам прямолинейных идеального и неидеального (неоднородного) стержней с параметром гибкости $\lambda=867$ при действии осевой сжимающей силы. В расчётной модели учтена геометрическая и физическая нелинейность, пластичность, изотропность, реальная диаграмма деформирования материала. Показано, что в идеальном стержне деформации обусловлены эффектом Пуассона, изгибных деформаций нет. Установлено, что для неидеального стержня существует критическая сила, при которой происходит потеря устойчивости стержня, связанная со значительными поперечными перемещениями (прогибами). Величина полученной критической силы согласуется с известным решением Эйлера.

Уравнения механики деформируемого твёрдого тела записываются, как правило, в соответствующей системе отсчёта - соответствующей системе координат. В зависимости от формы тела используются декартовы, полярные, цилиндрические, сферические [15-17] и другие координаты, например криволинейные подвижные лагранжевы [18], биполярные координаты [19]. Общие уравнения механики можно записать также и для общего случая произвольных криволинейных координат [1]. В данной работе используется наиболее часто применяемая в задачах, после декартовой, цилиндрическая система координат.

Цилиндрическая система координат используется при решении многих задач как линейной, так и нелинейной теории упругости. Так, в работе [20] выполнено исследование напряжённо-деформированного состояния пороупругого цилиндрического тела при радиальном равномерном сжатии, в результате чего построена математическая модель, описывающая неоднородное напряжённо-деформированное состояние цилиндрического тела для материалов с пористой структурой при упругой работе полностью сжатой матрицы. В [21] в цилиндрической системе координат рассматривается задача устойчивости кольцевых пластин с криволинейными структурами армирования в условиях плоского напряжённого состояния. Получен общий вид форм потери устойчивости, что позволило свести двумерную задачу к одномерной. Определены критические нагрузки для законов армирования по спирали Архимеда и логарифмической спирали. На основе метода малого параметра [22] построено аналитическое решение стохастической нелинейной краевой задачи установившейся ползучести для толстостенной трубы, находящейся под действием внутреннего давления в цилиндрических координатах. Приведено обобщение задачи расчёта толстостенной трубы, свойства материала которой описываются случайной функцией двух аргументов. В работе [23] в ци- 
линдрической системе координат рассмотрено напряжённое состояние пространства, заполненного сыпучим материалом. Микроструктура сыпучего материала обусловливает возможность объёмной пластической деформации за счёт деформации сдвига. Это подтверждается ассоциированным законом течения к условию пластичности, предполагающего зависимость предельного касательного напряжённого состояния от давления, что, в свою очередь, допускает возможность замкнутого вида условия пластичности в пространстве главных напряжений. В той же системе координат методом конечных элементов рассматриваются состояния толстостенного цилиндра при двух видах нагружения в неоднородном температурном поле [24]. При этом исследуется влияние учёта связанности численного определения напряжённо-деформированного состояния и температурного поля на решение задачи нелинейной термоупругости. Статья [25] посвящена упругопластическому кручению двухслойного слабоанизотропного стержня некругового поперечного сечения, представляющего собой двухсвязную область. Решение строится в предположении, что пластическая область целиком охватывает внешний контур поперечного сечения и существует упругопластическая граница, которая расположена между внутренним контуром и границей раздела слоёв. Задача решается в цилиндрической системе координат. В работе [26] так же в цилиндрической системе координат исследуется распространение нестационарных осесимметричных поверхностных возмущений в полупространстве, заполненном упругой однородной изотропной средой Коссера. Замкнутая система уравнений включает в себя уравнения относительно нетривиальных компонент потенциалов перемещения и угла поворота, а также соотношения, связывающие перемещения с потенциалами, и компонент тензоров напряжений и моментных напряжений с перемещениями и углом поворота. На граничной плоскости заданы нормальные перемещения. Показано, что учёт моментных напряжений приводит к качественным изменениям, а именно, наблюдается дополнительный волновой фронт. В статье [27] анализируются две анизотропные упругие модели, описывающие механическое поведение древесины: линейная ортотропная модель и цилиндрически ортотропная модель. Показано, что вторая модель является более сложной, но зато более адекватно описывает механические свойства древесины.

В статье [28] представлена разработка математической модели для описания механических свойств однослойных углеродных нанотрубок (ОСНТ). Связи углерод - углерод $(\mathrm{C}-\mathrm{C})$ между двумя соседними атомами смоделированы как балки Эйлера. Материальные константы балочного элемента - модуль Юнга и коэффициент Пуассона - определяются на атомном уровне. Расчёт выполнен в цилиндрических координатах. В данной работе строятся дифференциальные уравнения равновесия в перемещениях для случая плоского деформирования сплошной среды в цилиндрических координатах $u=u(r, \varphi), v=v(r, \varphi), w=0$ при аппроксимации замыкающих уравнений произвольной формы билинейными функциями без учёта геометрической нелинейности. Дифференциальные уравнения равновесия в перемещениях для случая плоского деформирования сплошной среды в декартовых координатах при аппроксимации замыкающих уравнений произвольной формы билинейными функциями без учёта и с учётом геометрической нелинейности приведены в работах [29, 30], а для случая осесимметричного деформирования сплошной среды при аппроксимации замыкающих уравнений произвольной формы билинейными функциями с учётом и без учёта геометрической нелиней- 
ности - в [31]. Дифференциальные уравнения равновесия в перемещениях для случая центрально-симметричного деформирования сплошной среды при аппроксимации замыкающих уравнений произвольной формы билинейными функциями с учётом и без учёта геометрической нелинейности разработаны в [32]. Вопросы аппроксимации диаграмм объёмного $\sigma=\sigma(\varepsilon)$ и сдвигового $T=T(\Gamma)$ деформирования при помощи билинейных функций, а также вопросы нахождения оптимального расположения точки излома на билинейном графике в [33].

\section{Построение физических уравнений}

В соответствии с рис. 1 и 2 секущие модули объёмного расширения (сжатия) $K=K(\varepsilon, \Gamma)$ и сдвига $G=G(\varepsilon, \Gamma)$ на первом прямолинейном участке диаграмм $\sigma=\sigma(\varepsilon)$ и $T=T(\Gamma)$ будут определяться выражениями

$$
\begin{gathered}
K=\frac{1}{3} K_{0}=\text { const } ; \\
G=G_{0}=\text { const } .
\end{gathered}
$$

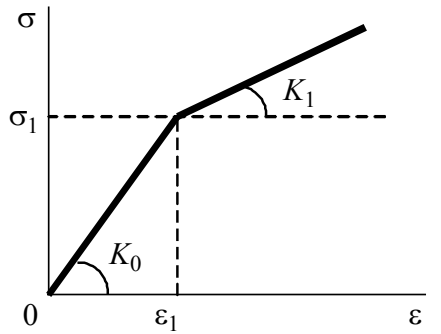

Рис. 1. Диаграмма $\sigma=\sigma(\varepsilon)$

Fig. 1. Curve $\sigma=\sigma(\varepsilon)$

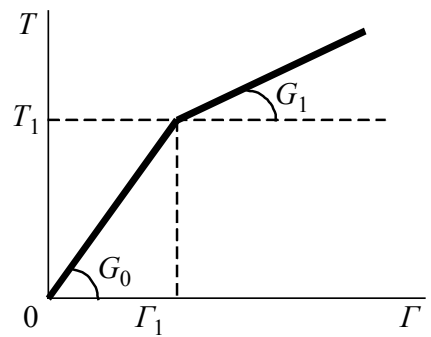

Pис. 2. Диаграмма $T=T(\Gamma)$

Fig. 2. Curve $T=T(\Gamma)$

На втором прямолинейном участке диаграмм $\sigma=\sigma(\varepsilon)$ и $T=T(\Gamma)$ секущий модуль объёмного расширения (сжатия) $K=K(\varepsilon, \Gamma)$ и секущий модуль сдвига $G=G(\varepsilon, \Gamma)$ будут определяться выражениями

$$
\begin{gathered}
K=K(\varepsilon)=\frac{1}{3}\left[K_{1}+\left(K_{0}-K_{1}\right) \frac{\varepsilon_{1}}{\varepsilon}\right] \neq \text { const } ; \\
G=G(\Gamma)=G_{1}+\left(G_{0}-G_{1}\right) \frac{\Gamma_{1}}{\Gamma} \neq \text { const } .
\end{gathered}
$$

Здесь $K_{0}$ - начальный модуль объёмного расширения (сжатия); $G_{0}-$ начальный модуль сдвига; $K_{1}-$ модуль упрочнения при объёмном расширении (сжатии); $G_{1}$ - модуль упрочнения при сдвиге; $\sigma$ - первый инвариант тензора напряжений; $\varepsilon-$ первый инвариант тензора деформаций; $T$ - интенсивность касательных напряжений; $\Gamma$ - интенсивность деформаций сдвига.

Так как построение билинейных диаграмм объёмного и сдвигового деформирования выполняется, вообще говоря, независимо друг от друга, а при плоской 
деформации - в цилиндрических координатах

$$
\begin{gathered}
\varepsilon=\varepsilon_{r r}+\varepsilon_{\varphi \varphi} \\
\Gamma=\sqrt{\frac{2}{3}} \sqrt{\left(\varepsilon_{r r}-\varepsilon_{\varphi \varphi}\right)^{2}+\varepsilon_{r r}^{2}+\varepsilon_{\varphi \varphi}^{2}+\frac{3}{2} \varepsilon_{r \varphi}^{2}},
\end{gathered}
$$

И

то установить связь между точками излома билинейных диаграмм $\sigma=\sigma(\varepsilon)$ и $T=T(\Gamma)$ в явном виде не представляется возможным.

Здесь

$$
\varepsilon_{r r}=\frac{\partial u}{\partial r} ; \quad \varepsilon_{\varphi \varphi}=\frac{1}{r}\left(\frac{\partial v}{\partial \varphi}+u\right) ; \quad \varepsilon_{r \varphi}=\varepsilon_{\varphi r}=\frac{\partial v}{\partial r}+\frac{1}{r} \frac{\partial u}{\partial \varphi}-\frac{v}{r}
$$

причём

$$
\begin{aligned}
& \frac{\partial \varepsilon_{r \varphi}}{\partial r}=\frac{\partial \varepsilon_{\varphi r}}{\partial r}=\frac{\partial^{2} v}{\partial r^{2}}-\frac{1}{r^{2}} \frac{\partial u}{\partial \varphi}+\frac{1}{r} \frac{\partial^{2} u}{\partial r \partial \varphi}-\frac{1}{r^{2}}\left(r \frac{\partial v}{\partial r}-v\right) ; \\
& \frac{\partial \varepsilon_{r \varphi}}{\partial \varphi}=\frac{\partial \varepsilon_{\varphi r}}{\partial \varphi}=\frac{\partial^{2} v}{\partial r \partial \varphi}+\frac{1}{r} \frac{\partial^{2} u}{\partial \varphi^{2}}-\frac{1}{r} \frac{\partial v}{\partial \varphi} ; \\
& \frac{\partial \varepsilon}{\partial r}=\frac{\partial \varepsilon_{r r}}{\partial r}+\frac{\partial \varepsilon_{\varphi \varphi}}{\partial r}=\frac{\partial^{2} u}{\partial r^{2}}+\left[-\frac{1}{r^{2}}\left(\frac{\partial v}{\partial \varphi}+u\right)+\frac{1}{r}\left(\frac{\partial^{2} v}{\partial r \partial \varphi}+\frac{\partial u}{\partial r}\right)\right] ; \\
& \frac{\partial \varepsilon}{\partial \varphi}=\frac{\partial \varepsilon_{r r}}{\partial \varphi}+\frac{\partial \varepsilon_{\varphi \varphi}}{\partial \varphi}=\frac{\partial^{2} u}{\partial r \partial \varphi}+\frac{1}{r}\left(\frac{\partial^{2} v}{\partial \varphi^{2}}+\frac{\partial u}{\partial \varphi}\right) ; \\
& \frac{\partial \Gamma}{\partial r}=\frac{2}{3 \Gamma}\left[\left(2 \varepsilon_{r r}-\varepsilon_{\varphi \varphi}\right) \frac{\partial \varepsilon_{r r}}{\partial r}+\left(2 \varepsilon_{\varphi \varphi}-\varepsilon_{r r}\right) \frac{\partial \varepsilon_{\varphi \varphi}}{\partial r}+\frac{3}{2} \frac{\partial \varepsilon_{r \varphi}}{\partial r} \varepsilon_{r \varphi}\right] ; \\
& \frac{\partial \Gamma}{\partial \varphi}=\frac{2}{3 \Gamma}\left[\left(2 \varepsilon_{r r}-\varepsilon_{\varphi \varphi}\right) \frac{\partial \varepsilon_{r r}}{\partial \varphi}+\left(2 \varepsilon_{\varphi \varphi}-\varepsilon_{r r}\right) \frac{\partial \varepsilon_{\varphi \varphi}}{\partial \varphi}+\frac{3}{2} \frac{\partial \varepsilon_{r \varphi}}{\partial \varphi} \varepsilon_{r \varphi}\right] .
\end{aligned}
$$

Рассмотрим шесть основных случаев физических зависимостей.

Случай 1: $K_{0} \neq K_{1}, G_{0} \neq G_{1}$. Здесь возможны три варианта взаимного расположения абсцисс точек излома билинейных диаграмм объёмного и сдвигового деформирования.

a). Численные значения абсцисс точек излома билинейных диаграмм совпадают, то есть $\left|\varepsilon_{1}\right|=\Gamma_{1}$. При этом $0 \leq|\varepsilon| \leq\left|\varepsilon_{1}\right|$ и $0 \leq \Gamma \leq \Gamma_{1}$.

б). Численные значения абсцисс точек излома билинейных диаграмм не совпадают, то есть $\left|\varepsilon_{1}\right|<\Gamma_{1}$. При этом $0 \leq|\varepsilon| \leq\left|\varepsilon_{1}\right|$ и $0 \leq \Gamma \leq \Gamma_{0}<\Gamma_{1}$. Здесь интенсивности $\Gamma_{0}$ соответствуют такие компоненты деформации, что $\left|\varepsilon_{r r}+\varepsilon_{\varphi \varphi}\right|=\left|\varepsilon_{1}\right|$.

в). Численные значения абсцисс точек излома билинейных диаграмм не совпадают, то есть $\left|\varepsilon_{1}\right|>\Gamma_{1}$. При этом $0 \leq|\varepsilon| \leq \varepsilon_{0}<\varepsilon_{1}$ и $0 \leq \Gamma \leq \Gamma_{1}$. Здесь объёмной деформации $\varepsilon_{0}$ соответствуют такие компоненты деформации, что

$$
\sqrt{\frac{2}{3}} \sqrt{\left(\varepsilon_{r r}-\varepsilon_{\varphi \varphi}\right)^{2}+\varepsilon_{r r}^{2}+\varepsilon_{\varphi \varphi}^{2}+\frac{3}{2} \varepsilon_{r \varphi}^{2}}=\Gamma_{1} .
$$


В этом случае физические уравнения плоской деформации в цилиндрических координатах с учётом формул (1) и (2) будут иметь вид

$$
\begin{aligned}
& \sigma_{r r}=\frac{1}{3} K_{0} \varepsilon+2 G_{0}\left(\varepsilon_{r r}-\frac{1}{3} \varepsilon\right) ; \\
& \sigma_{\varphi \varphi}=\frac{1}{3} K_{0} \varepsilon+2 G_{0}\left(\varepsilon_{\varphi \varphi}-\frac{1}{3} \varepsilon\right) ; \\
& \sigma_{r \varphi}=\sigma_{\varphi r}=G_{0} \varepsilon_{r \varphi}, \quad \sigma_{z z}=\left(\frac{1}{3} K_{0}-\frac{2}{3} G_{0}\right) \varepsilon .
\end{aligned}
$$

Случай 2: $K_{0} \neq K_{1}, G_{0} \neq G_{1}$. Численные значения абсцисс точек излома билинейных диаграмм не совпадают, то есть $\left|\varepsilon_{1}\right|<\Gamma_{1}$. При этом $\left|\varepsilon_{1}\right| \leq|\varepsilon| \leq \varepsilon_{0}$ и $\Gamma_{0} \leq \Gamma \leq \Gamma_{1}$. Здесь объёмной деформации $\varepsilon_{0}$ соответствуют такие компоненты деформации, что $\sqrt{\frac{2}{3}} \sqrt{\left(\varepsilon_{r r}-\varepsilon_{\varphi \varphi}\right)^{2}+\varepsilon_{r r}^{2}+\varepsilon_{\varphi \varphi}^{2}+\frac{3}{2} \varepsilon_{r \varphi}^{2}}=\Gamma_{1}$, а интенсивности $\Gamma_{0}$ соответствуют такие компоненты деформации, что $\left|\varepsilon_{r r}+\varepsilon_{\varphi \varphi}\right|=\left|\varepsilon_{1}\right|$.

В этом случае физические уравнения плоской деформации в цилиндрических координатах с учётом формул (2) и (3) будут иметь вид

$$
\begin{aligned}
& \sigma_{r r}=\frac{1}{3}\left[K_{1}+\left(K_{0}-K_{1}\right) \frac{\varepsilon_{1}}{\varepsilon}\right] \varepsilon+2 G_{0}\left(\varepsilon_{r r}-\frac{1}{3} \varepsilon\right) ; \\
& \sigma_{\varphi \varphi}=\frac{1}{3}\left[K_{1}+\left(K_{0}-K_{1}\right) \frac{\varepsilon_{1}}{\varepsilon}\right] \varepsilon+2 G_{0}\left(\varepsilon_{\varphi \varphi}-\frac{1}{3} \varepsilon\right) ; \\
& \sigma_{r \varphi}=\sigma_{\varphi r}=G_{0} \varepsilon_{r \varphi} ; \\
& \sigma_{z z}=\left\{\frac{1}{3}\left[K_{1}+\left(K_{0}-K_{1}\right) \frac{\varepsilon_{1}}{\varepsilon}\right]-\frac{2}{3} G_{0}\right\} \varepsilon .
\end{aligned}
$$

Случай 3: $K_{0} \neq K_{1}, G_{0} \neq G_{1}$. Численные значения абсцисс точек излома билинейных диаграмм не совпадают, то есть $\left|\varepsilon_{1}\right|>\Gamma_{1}$. При этом $\left|\varepsilon_{0}\right| \leq|\varepsilon| \leq\left|\varepsilon_{1}\right|$ и $\Gamma_{1} \leq \Gamma \leq \Gamma_{0}$. Здесь объёмной деформации $\varepsilon_{0}$ соответствуют такие компоненты деформации, что $\sqrt{\frac{2}{3}} \sqrt{\left(\varepsilon_{r r}-\varepsilon_{\varphi \varphi}\right)^{2}+\varepsilon_{r r}^{2}+\varepsilon_{\varphi \varphi}^{2}+\frac{3}{2} \varepsilon_{r \varphi}^{2}}=\Gamma_{1}$, а интенсивности $\Gamma_{0}-$ такие компоненты деформации, что $\left|\varepsilon_{r r}+\varepsilon_{\varphi \varphi}\right|=\left|\varepsilon_{1}\right|$.

В этом случае физические уравнения плоской деформации в цилиндрических координатах с учётом формул (1) и (4) будут иметь вид

$$
\begin{aligned}
& \sigma_{r r}=\frac{1}{3} K_{0} \varepsilon+2\left[G_{1}+\left(G_{0}-G_{1}\right) \frac{\Gamma_{1}}{\Gamma}\right]\left(\varepsilon_{r r}-\frac{1}{3} \varepsilon\right) ; \\
& \sigma_{\varphi \varphi}=\frac{1}{3} K_{0} \varepsilon+2\left[G_{1}+\left(G_{0}-G_{1}\right) \frac{\Gamma_{1}}{\Gamma}\right]\left(\varepsilon_{\varphi \varphi}-\frac{1}{3} \varepsilon\right) ; \\
& \sigma_{r \varphi}=\sigma_{\varphi r}=\left[G_{1}+\left(G_{0}-G_{1}\right) \frac{\Gamma_{1}}{\Gamma}\right] \varepsilon_{r \varphi} ; \\
& \sigma_{z z}=\left\{\frac{1}{3} K_{0}-\frac{2}{3}\left[G_{1}+\left(G_{0}-G_{1}\right) \frac{\Gamma_{1}}{\Gamma}\right]\right\} \varepsilon .
\end{aligned}
$$


Случай 4: $K_{0} \neq K_{1}, G_{0} \neq G_{1}$. Здесь возможны три варианта взаимного расположения абсцисс точек излома билинейных диаграмм объёмного и сдвигового деформирования.

а). Численные значения абсцисс точек излома билинейных диаграмм совпадают, то есть $\left|\varepsilon_{1}\right|=\Gamma_{1}$. При этом $|\varepsilon| \geq\left|\varepsilon_{1}\right|$ и $\Gamma \geq \Gamma_{1}$.

б). Численные значения абсцисс точек излома билинейных диаграмм не совпадают, то есть $\left|\varepsilon_{1}\right|<\Gamma_{1}$. При этом $|\varepsilon| \geq\left|\varepsilon_{0}\right|$ и $\Gamma \geq \Gamma_{1}$. Здесь объёмной деформации $\varepsilon_{0}$ соответствуют такие компоненты деформации, что

$$
\sqrt{\frac{2}{3}} \sqrt{\left(\varepsilon_{r r}-\varepsilon_{\varphi \varphi}\right)^{2}+\varepsilon_{r r}^{2}+\varepsilon_{\varphi \varphi}^{2}+\frac{3}{2} \varepsilon_{r \varphi}^{2}}=\Gamma_{1} .
$$

в). Численные значения абсцисс точек излома билинейных диаграмм не совпадают, то есть $\left|\varepsilon_{1}\right|>\Gamma_{1}$. При этом $|\varepsilon| \geq\left|\varepsilon_{1}\right|$ и $\Gamma \geq \Gamma_{0}$. Здесь интенсивности $\Gamma_{0}$ соответствуют такие компоненты деформации, что $\left|\varepsilon_{r r}+\varepsilon_{\varphi \varphi}\right|=\left|\varepsilon_{1}\right|$.

В этом случае физические уравнения плоской деформации в цилиндрических координатах с учётом формул (2) и (4) будут иметь вид

$$
\begin{aligned}
& \sigma_{r r}=\frac{1}{3}\left[K_{1}+\left(K_{0}-K_{1}\right) \frac{\varepsilon_{1}}{\varepsilon}\right] \varepsilon+2\left[G_{1}+\left(G_{0}-G_{1}\right) \frac{\Gamma_{1}}{\Gamma}\right]\left(\varepsilon_{r r}-\frac{1}{3} \varepsilon\right) ; \\
& \sigma_{\varphi \varphi}=\frac{1}{3}\left[K_{1}+\left(K_{0}-K_{1}\right) \frac{\varepsilon_{1}}{\varepsilon}\right] \varepsilon+2\left[G_{1}+\left(G_{0}-G_{1}\right) \frac{\Gamma_{1}}{\Gamma}\right]\left(\varepsilon_{\varphi \varphi}-\frac{1}{3} \varepsilon\right) ; \\
& \sigma_{r \varphi}=\sigma_{\varphi r}=\left[G_{1}+\left(G_{0}-G_{1}\right) \frac{\Gamma_{1}}{\Gamma}\right] \varepsilon_{r \varphi} ; \\
& \sigma_{z z}=\left\{\frac{1}{3}\left[K_{1}+\left(K_{0}-K_{1}\right) \frac{\varepsilon_{1}}{\varepsilon}\right]-\frac{2}{3}\left[G_{1}+\left(G_{0}-G_{1}\right) \frac{\Gamma_{1}}{\Gamma}\right]\right\} \varepsilon .
\end{aligned}
$$

Случай 5: $K_{0}=K_{1}, G_{0} \neq G_{1}$. Точка излома на диаграмме $\sigma=\sigma(\varepsilon)$ отсутствует. При этом, если $0 \leq \Gamma \leq \Gamma_{1}$, то физические уравнения плоской деформации в цилиндрических координатах будут иметь вид (5); если $\Gamma \geq \Gamma_{1}$, то физические уравнения плоской деформации будут иметь вид (7).

Случай 6: $K_{0} \neq K_{1}, G_{0}=G_{1}$. Точка излома на диаграмме $T=T(\Gamma)$ отсутствует. При этом, если $0 \leq|\varepsilon| \leq\left|\varepsilon_{1}\right|$, то физические уравнения плоской деформации в цилиндрических координатах будут иметь вид (5); если $|\varepsilon| \geq\left|\varepsilon_{1}\right|$, то физические уравнения плоской деформации будут иметь вид (6).

\section{Построение дифференциальных уравнений равновесия}

Подставляя физические уравнения (5) - (8) в дифференциальные уравнения равновесия плоской деформации сплошной среды в цилиндрических координатах [1]:

$$
\left.\begin{array}{c}
\frac{\partial \sigma_{r r}}{\partial r}+\frac{1}{r} \frac{\partial \sigma_{r \varphi}}{\partial \varphi}+\frac{\sigma_{r r}-\sigma_{\varphi \varphi}}{r}+F_{r}=0 \\
\frac{\partial \sigma_{\varphi r}}{\partial r}+\frac{1}{r} \frac{\partial \sigma_{\varphi \varphi}}{\partial \varphi}+\frac{2}{r} \sigma_{r \varphi}+F_{\varphi}=0
\end{array}\right\}
$$

получим четыре вида разрешающих уравнений в перемещениях, имеющих одну и 
ту же структуру:

$$
\left\{\begin{array}{l}
A_{1} \frac{\partial^{2} u}{\partial r^{2}}+B_{1} \frac{\partial^{2} u}{\partial \varphi^{2}}+C_{1} \frac{\partial^{2} u}{\partial r \partial \varphi}+D_{1} \frac{\partial^{2} v}{\partial r^{2}}+E_{1} \frac{\partial^{2} v}{\partial \varphi^{2}}+F_{1} \frac{\partial^{2} v}{\partial r \partial \varphi}+A+F_{r}=0 \\
A_{2} \frac{\partial^{2} u}{\partial r^{2}}+B_{2} \frac{\partial^{2} u}{\partial \varphi^{2}}+C_{2} \frac{\partial^{2} u}{\partial r \partial \varphi}+D_{2} \frac{\partial^{2} v}{\partial r^{2}}+E_{2} \frac{\partial^{2} v}{\partial \varphi^{2}}+F_{2} \frac{\partial^{2} v}{\partial r \partial \varphi}+B+F_{\varphi}=0
\end{array}\right.
$$

Коэффициенты $A_{1}, B_{1}, C_{1}, D_{1}, E_{1}, F_{1}, A$ и $A_{2}, B_{2}, C_{2}, D_{2}, E_{2}, F_{2}, B$ в уравнениях (10) зависят от вида физических уравнений.

1) Для физических уравнений (5) получим

$$
\begin{aligned}
& A_{1}=\frac{1}{3} K_{0}+\frac{4}{3} G_{0} ; B_{1}=G_{0} \frac{1}{r^{2}} ; C_{1}=0 ; D_{1}=0 ; E_{1}=0 ; F_{1}=\left(\frac{1}{3} K_{0}+\frac{1}{3} G_{0}\right) \frac{1}{r} ; \\
& A=\left(\frac{1}{3} K_{0}+\frac{4}{3} G_{0}\right) \frac{1}{r}\left(\frac{\partial u}{\partial r}-\frac{u}{r}\right)-\left(\frac{1}{3} K_{0}+\frac{7}{3} G_{0}\right) \frac{1}{r^{2}} \frac{\partial v}{\partial \varphi} ; \\
& A_{2}=0 ; \quad B_{2}=0 ; C_{2}=\left(\frac{1}{3} K_{0}+\frac{1}{3} G_{0}\right) \frac{1}{r} ; D_{2}=G_{0} ; E_{2}=\left(\frac{1}{3} K_{0}+\frac{4}{3} G_{0}\right) \frac{1}{r^{2}} ; F_{2}=0 ; \\
& B=G_{0} \frac{1}{r}\left(\frac{\partial v}{\partial r}-\frac{v}{r}\right)+\left(\frac{1}{3} K_{0}+\frac{7}{3} G_{0}\right) \frac{1}{r^{2}} \frac{\partial u}{\partial \varphi} .
\end{aligned}
$$

2) Для физических уравнений (6)

$$
\begin{aligned}
& A_{1}=\frac{1}{3} K_{1}+\frac{4}{3} G_{0} ; B_{1}=G_{0} \frac{1}{r^{2}} ; C_{1}=0 ; D_{1}=0 ; E_{1}=0 ; F_{1}=\left(\frac{1}{3} K_{1}+\frac{1}{3} G_{0}\right) \frac{1}{r} ; \\
& A=\left(\frac{1}{3} K_{1}+\frac{4}{3} G_{0}\right) \frac{1}{r}\left(\frac{\partial u}{\partial r}-\frac{u}{r}\right)-\left(\frac{1}{3} K_{1}+\frac{7}{3} G_{0}\right) \frac{1}{r^{2}} \frac{\partial v}{\partial \varphi} ; \\
& A_{2}=0 ; B_{2}=0 ; C_{2}=\left(\frac{1}{3} K_{1}+\frac{1}{3} G_{0}\right) \frac{1}{r} ; D_{2}=G_{0} ; E_{2}=\left(\frac{1}{3} K_{1}+\frac{4}{3} G_{0}\right) \frac{1}{r^{2}} ; F_{2}=0 ; \\
& B=G_{0} \frac{1}{r}\left(\frac{\partial v}{\partial r}-\frac{v}{r}\right)+\left(\frac{1}{3} K_{1}+\frac{7}{3} G_{0}\right) \frac{1}{r^{2}} \frac{\partial u}{\partial \varphi} .
\end{aligned}
$$

3) Для физических уравнений (7)

$$
\begin{aligned}
A_{1} & =\frac{1}{3} K_{0}+\frac{4}{3} G_{1}+\frac{4}{3}\left(G_{0}-G_{1}\right) \frac{\Gamma_{1}}{\Gamma}-\frac{4}{3}\left(G_{0}-G_{1}\right) \frac{\Gamma_{1}}{\Gamma^{3}}\left(\varepsilon_{r r}-\frac{1}{3} \varepsilon\right)\left(2 \varepsilon_{r r}-\varepsilon_{\varphi \varphi}\right) ; \\
B_{1} & =\left[G_{1}+\left(G_{0}-G_{1}\right) \frac{\Gamma_{1}}{\Gamma}-\left(G_{0}-G_{1}\right) \varepsilon_{r \varphi}^{2} \frac{\Gamma_{1}}{\Gamma^{3}}\right] \frac{1}{r^{2}} ; \\
C_{1} & =-2\left(G_{0}-G_{1}\right) \varepsilon_{r \varphi} \frac{\Gamma_{1}}{\Gamma^{3}}\left[\left(\varepsilon_{r r}-\frac{1}{3} \varepsilon\right)+\frac{1}{3}\left(2 \varepsilon_{r r}-\varepsilon_{\varphi \varphi}\right)\right] \frac{1}{r} ; \\
D_{1} & =-2\left(G_{0}-G_{1}\right) \varepsilon_{r \varphi} \frac{\Gamma_{1}}{\Gamma^{3}}\left(\varepsilon_{r r}-\frac{1}{3} \varepsilon\right) ; \\
E_{1} & =-\frac{2}{3}\left(G_{0}-G_{1}\right) \varepsilon_{r \varphi} \frac{\Gamma_{1}}{\Gamma^{3}}\left(2 \varepsilon_{\varphi \varphi}-\varepsilon_{r r}\right) \frac{1}{r^{2}} ; \\
F_{1} & =\left[\frac{1}{3} K_{0}-\frac{2}{3} G_{1}-\frac{2}{3}\left(G_{0}-G_{1}\right) \frac{\Gamma_{1}}{\Gamma}-\frac{4}{3}\left(G_{0}-G_{1}\right) \frac{\Gamma_{1}}{\Gamma^{3}}\left(\varepsilon_{r r}-\frac{1}{3} \varepsilon\right)\left(2 \varepsilon_{\varphi \varphi}-\varepsilon_{r r}\right)\right] \frac{1}{r}+ \\
& +\left[G_{1}+\left(G_{0}-G_{1}\right) \frac{\Gamma_{1}}{\Gamma}-\left(G_{0}-G_{1}\right) \frac{\Gamma_{1}}{\Gamma^{3}} \varepsilon_{r \varphi}^{2}\right] \frac{1}{r} ;
\end{aligned}
$$




$$
\begin{aligned}
& A=\left[\frac{1}{3} K_{0}-\frac{2}{3} G_{1}-\frac{2}{3}\left(G_{0}-G_{1}\right) \frac{\Gamma_{1}}{\Gamma}-\frac{4}{3}\left(G_{0}-G_{1}\right) \frac{\Gamma_{1}}{\Gamma^{3}}\left(\varepsilon_{r r}-\frac{1}{3} \varepsilon\right)\left(2 \varepsilon_{\varphi \varphi}-\varepsilon_{r r}\right)\right] \times \\
& \times \frac{1}{r}\left(\frac{\partial u}{\partial r}-\frac{1}{r} \frac{\partial v}{\partial \varphi}-\frac{u}{r}\right)+2\left(G_{0}-G_{1}\right) \varepsilon_{r \varphi} \frac{\Gamma_{1}}{\Gamma^{3}}\left(\varepsilon_{r r}-\frac{1}{3} \varepsilon\right) \frac{1}{r}\left(\frac{1}{r} \frac{\partial u}{\partial \varphi}+\frac{\partial v}{\partial r}-\frac{v}{r}\right)- \\
& -\frac{2}{3}\left(G_{0}-G_{1}\right) \frac{\Gamma_{1}}{\Gamma^{3}} \varepsilon_{r \varphi}\left(2 \varepsilon_{\varphi \varphi}-\varepsilon_{r r}\right) \frac{1}{r^{2}} \frac{\partial u}{\partial \varphi}-\left[G_{1}+\left(G_{0}-G_{1}\right) \frac{\Gamma_{1}}{\Gamma}-\left(G_{0}-G_{1}\right) \frac{\Gamma_{1}}{\Gamma^{3}} \varepsilon_{r \varphi}^{2}\right] \times \\
& \times \frac{1}{r^{2}} \frac{\partial v}{\partial \varphi}+\frac{2}{r}\left[G_{1}+\left(G_{0}-G_{1}\right) \frac{\Gamma_{1}}{\Gamma}\right]\left(\varepsilon_{r r}-\varepsilon_{\varphi \varphi}\right) \\
& A_{2}=-\frac{2}{3}\left(G_{0}-G_{1}\right) \varepsilon_{r \varphi} \frac{\Gamma_{1}}{\Gamma^{3}}\left(2 \varepsilon_{r r}-\varepsilon_{\varphi \varphi}\right) \text {; } \\
& B_{2}=-2\left(G_{0}-G_{1}\right) \varepsilon_{r \varphi} \frac{\Gamma_{1}}{\Gamma^{3}}\left(\varepsilon_{\varphi \varphi}-\frac{1}{3} \varepsilon\right) \frac{1}{r^{2}} \\
& C_{2}=\left[\frac{1}{3} K_{0}+\frac{1}{3} G_{1}+\frac{1}{3}\left(G_{0}-G_{1}\right) \frac{\Gamma_{1}}{\Gamma}-\frac{4}{3}\left(G_{0}-G_{1}\right) \frac{\Gamma_{1}}{\Gamma^{3}}\left(\varepsilon_{\varphi \varphi}-\frac{1}{3} \varepsilon\right)\left(2 \varepsilon_{r r}-\varepsilon_{\varphi \varphi}\right)-\left(G_{0}-G_{1}\right) \varepsilon_{r \varphi}^{2} \frac{\Gamma_{1}}{\Gamma^{3}}\right] \frac{1}{r} ; \\
& D_{2}=G_{1}+\left(G_{0}-G_{1}\right) \frac{\Gamma_{1}}{\Gamma}-\left(G_{0}-G_{1}\right) \varepsilon_{r \varphi}^{2} \frac{\Gamma_{1}}{\Gamma^{3}} \\
& E_{2}=\left[\frac{1}{3} K_{0}+\frac{4}{3} G_{1}+\frac{4}{3}\left(G_{0}-G_{1}\right) \frac{\Gamma_{1}}{\Gamma}-\frac{4}{3}\left(G_{0}-G_{1}\right) \frac{\Gamma_{1}}{\Gamma^{3}}\left(\varepsilon_{\varphi \varphi}-\frac{1}{3} \varepsilon\right)\left(2 \varepsilon_{\varphi \varphi}-\varepsilon_{r r}\right)\right] \frac{1}{r^{2}} ; \\
& F_{2}=-2\left(G_{0}-G_{1}\right) \varepsilon_{r \varphi} \frac{\Gamma_{1}}{\Gamma^{3}}\left[\frac{1}{3}\left(2 \varepsilon_{\varphi \varphi}-\varepsilon_{r r}\right)+\left(\varepsilon_{\varphi \varphi}-\frac{1}{3} \varepsilon\right)\right] \frac{1}{r} \text {; } \\
& B=\frac{1}{3} K_{0}+\frac{4}{3} G_{1}+\frac{4}{3}\left(G_{0}-G_{1}\right) \frac{\Gamma_{1}}{\Gamma}-\frac{4}{3}\left(G_{0}-G_{1}\right) \frac{\Gamma_{1}}{\Gamma^{3}}\left(\varepsilon_{\varphi \varphi}-\frac{1}{3} \varepsilon\right)\left(2 \varepsilon_{\varphi \varphi}-\varepsilon_{r r}\right) \frac{1}{r^{2}} \frac{\partial u}{\partial \varphi}- \\
& -\frac{2}{3}\left(G_{0}-G_{1}\right) \varepsilon_{r \varphi} \frac{\Gamma_{1}}{\Gamma^{3}}\left(2 \varepsilon_{\varphi \varphi}-\varepsilon_{r r}\right) \frac{1}{r}\left(\frac{\partial u}{\partial r}-\frac{1}{r} \frac{\partial v}{\partial \varphi}-\frac{u}{r}\right)+2\left(G_{0}-G_{1}\right) \varepsilon_{r \varphi} \frac{\Gamma_{1}}{\Gamma^{3}}\left(\varepsilon_{\varphi \varphi}-\frac{1}{3} \varepsilon\right) \frac{1}{r^{2}} \frac{\partial v}{\partial \varphi}- \\
& -\left[G_{1}+\left(G_{0}-G_{1}\right) \frac{\Gamma_{1}}{\Gamma}-\left(G_{0}-G_{1}\right) \frac{\Gamma_{1}}{\Gamma^{3}} \varepsilon_{r \varphi}^{2}\right]\left(\frac{1}{r} \frac{\partial u}{\partial \varphi}-\frac{\partial v}{\partial r}+\frac{v}{r}\right) \frac{1}{r}+\frac{2}{r}\left[G_{1}+\left(G_{0}-G_{1}\right) \frac{\Gamma_{1}}{\Gamma}\right] \varepsilon_{r \varphi} . \\
& \text { 4) Для физических уравнений (8) } \\
& A_{1}=\frac{1}{3} K_{1}+\frac{4}{3} G_{1}+\frac{4}{3}\left(G_{0}-G_{1}\right) \frac{\Gamma_{1}}{\Gamma}-\frac{4}{3}\left(G_{0}-G_{1}\right) \frac{\Gamma_{1}}{\Gamma^{3}}\left(\varepsilon_{r r}-\frac{1}{3} \varepsilon\right)\left(2 \varepsilon_{r r}-\varepsilon_{\varphi \varphi}\right) ; \\
& B_{1}=\left[G_{1}+\left(G_{0}-G_{1}\right) \frac{\Gamma_{1}}{\Gamma}-\left(G_{0}-G_{1}\right) \frac{\Gamma_{1}}{\Gamma^{3}} \varepsilon_{r \varphi}^{2}\right] \frac{1}{r^{2}} \text {; } \\
& C_{1}=-2\left(G_{0}-G_{1}\right) \varepsilon_{r \varphi} \frac{\Gamma_{1}}{\Gamma^{3}}\left[\left(\varepsilon_{r r}-\frac{1}{3} \varepsilon\right)+\frac{1}{3}\left(2 \varepsilon_{r r}-\varepsilon_{\varphi \varphi}\right)\right] \frac{1}{r} ; \\
& D_{1}=-2\left(G_{0}-G_{1}\right) \varepsilon_{r \varphi} \frac{\Gamma_{1}}{\Gamma^{3}}\left(\varepsilon_{r r}-\frac{1}{3} \varepsilon\right) \text {; } \\
& E_{1}=-\frac{2}{3}\left(G_{0}-G_{1}\right) \varepsilon_{r \varphi} \frac{\Gamma_{1}}{\Gamma^{3}}\left(2 \varepsilon_{\varphi \varphi}-\varepsilon_{r r}\right) \frac{1}{r^{2}} \text {; } \\
& F_{1}=\left[\frac{1}{3} K_{1}-\frac{2}{3} G_{1}-\frac{2}{3}\left(G_{0}-G_{1}\right) \frac{\Gamma_{1}}{\Gamma}-\frac{4}{3}\left(G_{0}-G_{1}\right) \frac{\Gamma_{1}}{\Gamma^{3}}\left(\varepsilon_{r r}-\frac{1}{3} \varepsilon\right)\left(2 \varepsilon_{\varphi \varphi}-\varepsilon_{r r}\right)\right] \frac{1}{r}+ \\
& +\left[G_{1}+\left(G_{0}-G_{1}\right) \frac{\Gamma_{1}}{\Gamma}-\left(G_{0}-G_{1}\right) \varepsilon_{r \varphi}^{2} \frac{\Gamma_{1}}{\Gamma^{3}}\right] \frac{1}{r} ;
\end{aligned}
$$




$$
\begin{aligned}
A= & {\left[\frac{1}{3} K_{1}-\frac{2}{3} G_{1}-\frac{2}{3}\left(G_{0}-G_{1}\right) \frac{\Gamma_{1}}{\Gamma}-\frac{4}{3}\left(G_{0}-G_{1}\right) \frac{\Gamma_{1}}{\Gamma^{3}}\left(\varepsilon_{r r}-\frac{1}{3} \varepsilon\right)\left(2 \varepsilon_{\varphi \varphi}-\varepsilon_{r r}\right)\right] \frac{1}{r}\left(\frac{\partial u}{\partial r}-\frac{1}{r} \frac{\partial v}{\partial \varphi}-\frac{u}{r}\right)+} \\
& +2\left(G_{0}-G_{1}\right) \varepsilon_{r \varphi} \frac{\Gamma_{1}}{\Gamma^{3}}\left(\varepsilon_{r r}-\frac{1}{3} \varepsilon\right) \frac{1}{r}\left(\frac{1}{r} \frac{\partial u}{\partial \varphi}+\frac{\partial v}{\partial r}-\frac{v}{r}\right)-\frac{2}{3}\left(G_{0}-G_{1}\right) \varepsilon_{r \varphi} \frac{\Gamma_{1}}{\Gamma^{3}}\left(2 \varepsilon_{\varphi \varphi}-\varepsilon_{r r}\right) \frac{1}{r^{2}} \frac{\partial u}{\partial \varphi}- \\
& -\left[G_{1}+\left(G_{0}-G_{1}\right) \frac{\Gamma_{1}}{\Gamma}-\left(G_{0}-G_{1}\right) \varepsilon_{r \varphi}^{2} \frac{\Gamma_{1}}{\Gamma^{3}}\right] \frac{1}{r^{2}} \frac{\partial v}{\partial \varphi}+\frac{2}{r}\left[G_{1}+\left(G_{0}-G_{1}\right) \frac{\Gamma_{1}}{\Gamma}\right]\left(\varepsilon_{r r}-\varepsilon_{\varphi \varphi}\right) ; \\
A_{2}= & -\frac{2}{3}\left(G_{0}-G_{1}\right) \varepsilon_{r \varphi} \frac{\Gamma_{1}}{\Gamma^{3}}\left(2 \varepsilon_{r r}-\varepsilon_{\varphi \varphi}\right) ; \\
B_{2}= & -2\left(G_{0}-G_{1}\right) \varepsilon_{r \varphi} \frac{\Gamma_{1}}{\Gamma^{3}}\left(\varepsilon_{\varphi \varphi}-\frac{1}{3} \varepsilon\right) \frac{1}{r^{2}} ; \\
C_{2}= & {\left[\frac{1}{3} K_{1}+\frac{1}{3} G_{1}+\frac{1}{3}\left(G_{0}-G_{1}\right) \frac{\Gamma_{1}}{\Gamma}-\left(G_{0}-G_{1}\right) \varepsilon_{r \varphi}^{2} \frac{\Gamma_{1}}{\Gamma^{3}}-\frac{4}{3}\left(G_{0}-G_{1}\right) \frac{\Gamma_{1}}{\Gamma^{3}}\left(\varepsilon_{\varphi \varphi}-\frac{1}{3} \varepsilon\right)\left(2 \varepsilon_{r r}-\varepsilon_{\varphi \varphi}\right)\right] } \\
D_{2}= & G_{1}+\left(G_{0}-G_{1}\right) \frac{\Gamma_{1}}{\Gamma}-\left(G_{0}-G_{1}\right) \varepsilon_{r \varphi}^{2} \frac{\Gamma_{1}}{\Gamma^{3}} ; \\
E_{2}= & {\left[\frac{1}{3} K_{1}+\frac{4}{3} G_{1}+\frac{4}{3}\left(G_{0}-G_{1}\right) \frac{\Gamma_{1}}{\Gamma}-\frac{4}{3}\left(G_{0}-G_{1}\right) \frac{\Gamma_{1}}{\Gamma^{3}}\left(\varepsilon_{\varphi \varphi}-\frac{1}{3} \varepsilon\right)\left(2 \varepsilon_{\varphi \varphi}-\varepsilon_{r r}\right)\right] \frac{1}{r^{2}} } \\
& +\frac{4}{3}\left(G_{0}-G_{1}\right) \frac{\Gamma_{1}}{\Gamma^{3}}\left(\varepsilon_{\varphi \varphi}-\frac{1}{3} \varepsilon\right)\left(2 \varepsilon_{\varphi \varphi}-\varepsilon_{r r}\right) \frac{1}{r^{2}} \frac{\partial u}{\partial \varphi}+2\left(G_{0}-G_{1}\right) \varepsilon_{r \varphi} \frac{\Gamma_{1}}{\Gamma^{3}}\left(\varepsilon_{\varphi \varphi}-\frac{1}{3} \varepsilon\right) \frac{1}{r^{2}} \frac{\partial v}{\partial \varphi} \\
F_{2}= & -2\left(G_{0}-G_{1}\right) \varepsilon_{r \varphi} \frac{\Gamma_{1}}{\Gamma^{3}}\left[\frac{1}{3}\left(2 \varepsilon_{\varphi \varphi}-\varepsilon_{r r}\right)+\left(\varepsilon_{\varphi \varphi}-\frac{1}{3} \varepsilon\right)\right] \frac{1}{r} ; \\
B= & \frac{1}{3} K_{1}+\frac{4}{3} G_{1}+\frac{4}{3}\left(G_{0}-G_{1}\right) \frac{\Gamma_{1}}{\Gamma}-\frac{2}{3}\left(G_{0}-G_{1}\right) \varepsilon_{r \varphi} \frac{\Gamma_{1}}{\Gamma^{3}}\left(2 \varepsilon_{\varphi \varphi}-\varepsilon_{r r}\right) \frac{1}{r}\left(\frac{\partial u}{\partial r}-\frac{1}{r} \frac{\partial v}{\partial \varphi}-\frac{u}{r}\right)- \\
& {\left[G_{0}-G_{1}\right) \frac{\Gamma_{1}}{\Gamma}-\left(G_{0}-G_{1}\right) \varepsilon_{r \varphi}^{2} \frac{\Gamma_{1}}{\Gamma^{3}}\left(\frac{1}{r} \frac{\partial u}{\partial}-\frac{\partial v}{\partial r}+\frac{v}{r}\right) \frac{2}{r}\left[G_{1}+\left(G_{0}-G_{1}\right) \frac{\Gamma_{1}}{\Gamma}\right] \varepsilon_{r \varphi}+}
\end{aligned}
$$

В формулах (11) - (14)

$$
\begin{gathered}
\Gamma=\sqrt{\frac{2}{3}} \sqrt{\left(\varepsilon_{r r}-\varepsilon_{\varphi \varphi}\right)^{2}+\varepsilon_{r r}^{2}+\varepsilon_{\varphi \varphi}^{2}+\frac{3}{2} \varepsilon_{r \varphi}^{2}}= \\
=\sqrt{\frac{2}{3}} \sqrt{\left(\frac{\partial u}{\partial r}-\frac{1}{r} \frac{\partial v}{\partial \varphi}-\frac{u}{r}\right)^{2}+\left(\frac{\partial u}{\partial r}\right)^{2}+\frac{1}{r^{2}}\left(\frac{\partial v}{\partial \varphi}+u\right)^{2}+\frac{3}{2}\left(\frac{\partial v}{\partial r}+\frac{1}{r} \frac{\partial u}{\partial \varphi}-\frac{v}{r}\right)^{2}} .
\end{gathered}
$$

Таким образом, дифференциальные уравнения равновесия в перемещениях для плоской деформации сплошной среды в цилиндрических координатах при билинейной аппроксимации физических соотношений построены.

\section{Заключение}

Построенные в статье дифференциальные уравнения равновесия в перемещениях в цилиндрических координатах могут найти применение при определении напряжённо-деформированного состояния сплошных сред, находящихся в условиях плоской деформации, физические соотношения для которых аппроксимированы билинейными функциями. 
Замечание: предложенный в статье метод построения дифференциальных уравнений равновесия в перемещениях для геометрически линейных сплошных сред, физические соотношения для которых аппроксимированы билинейными функциями, может быть с успехом применён и для сплошных сред, механическое поведение которых описывается геометрически нелинейными моделями.

\section{ЛИТЕРАТУРА}

1. Новожилов В.В. Теория упругости. Л.: Судпромгиз, 1958. 370 с.

2. Бакушев С.В. Геометрически и физически нелинейная механика сплошной среды: Плоская задача. М.: Книжный дом «Либроком», 2013. 312 с.

3. Pудыx О.Л. Практические вопросы аппроксимации экспериментальных кривых степенными и дробно-линейными функциями // Вестник Томского государственного архитектурно-строительного университета. 2010. № 1 (26). С. 110-122.

4. Гоник Е.Г., Кибеи А.И., Петров М.В., Федорова Т.Г., Фролова И.А. Влияние аппроксимации диаграммы деформирования на критические нагрузки при поперечном изгибе цилиндрической оболочки // Проблемы прочности и пластичности. 2017. Т. 79. № 2. С. 169-181.

5. Король Е.3. Определение диаграмм нелинейного деформирования разносопротивляющихся материалов при неоднородном напряжённо-деформированном состоянии // Депонированная рукопись № 86-В2005 21.01.2005.

6. Зубчанинов В.Г. Общая математическая теория пластичности и постулаты макроскопической определимости и изотропии А.А. Ильюшина // Вестник Московского университета. Серия 1: Математика. Механика. 2018. № 5. С. 29-46.

7. Садовский В.M. Реологические модели разномодульных и сыпучих сред // Дальневосточный математический журнал. 2003. Т. 4. № 2. С. 252-263.

8. Морщинина A.A. Нелинейная осесимметричная задача теории упругости для полой сферы // Вестник Санкт-Петербургского университета. Математика. Механика. Астрономия. 2009. № 4. С. 84-88.

9. Федотова И.А. Математическая модель работы нелинейного стержневого элемента конструкции // Известия Петербургского университета путей сообщения. 2004. № 1. C. 7-12.

10. Мицкевич С.А., Крысько А.В., Жигалов М.В., Крысько В.А. Динамическая устойчивость пологих оболочек на прямоугольном плане с учётом геометрической и физической нелинейности // Проблемы прочности и пластичности. 2017. Т. 79. № 3. С. 249-258.

11. Махметова Н.М. Об одном алгоритме нелинейных задач статики подземных сооружений // Вестник Казахской академии транспорта и коммуникаций им. М. Тынышпаева. 2014. № 5 (90). С. $80-87$.

12. Крысько В.А., Папкова И.В., Салтыкова О.А., Бабенкова Т.В., Кашубина А.А. Сложные колебания балок Эйлера - Бернулли с учётом геометрической и физической нелинейностей // Международный научно-исследовательский журнал. 2014. № 3-1 (22). С. 14-16.

13. Петров B.B. Расчёт неоднородных по толщине оболочек с учётом физической и геометрической нелинейностей // Academia. Архитектура и строительство. 2016. № 1. C. $112-117$.

14. Егоров А.B. Деформирование центрально-сжатого гибкого стержня // Инженерный журнал: наука и инновации. 2018. № 4 (76). С. 1. DOI: 10.18698/2308-6033-2018-4-1750.

15. Лурье А.И. Нелинейная теория упругости. М.: Наука, 1980. 512 с.

16. Демидов С.П. Теория упругости: учебник для вузов. М.: Высш. школа, 1979. 432 с.

17. Папкович П.Ф. Теория упругости. М.; Л.: Оборонгиз, 1939. 643 с.

18. Зарипов Р.М., Масалимов Р.Б., Лисин Ю.В. Моделирование напряжённо-деформированного состояния прямолинейных и криволинейных участков трубопровода // Нефтегазовое дело. 2015. Т. 13. № 3. С. 103-109 с.

19. Стрельников В.Н., Суков Г.С., Волошин А.И., Чибисов Ю.В., Лесняк Г.А. Уравнения упругости в биполярных координатах // Прогресивні технології і системи машинобудування. 2010. № 2 (40). С. 248-253. 
20. Бунтов А.Е., Гоиев Д.В. Неоднородное напряжённо-деформированное состояние упругого цилиндрического тела с учётом внутренней структуры материала // Вестник Дагестанского государственного технического университета. Технические науки. 2018. T. 45. № 1. C. $8-21$.

21. Немировский Ю.В., Матвеев К.А., Моховнев Д.В. Рациональное проектирование армированных кольцевых пластин // Проблемы оптимального проектирования сооружений: сборник докладов 3-й Всероссийской конференции. Новосибирский ГАСУ (Сибстрин), Сибирское отделение Российской академии архитектуры и строительных наук, Сибирское отделение международной академии наук высшей школы. 2014. С. 272-282.

22. Радченко В.П., Попов Н.Н. Использование метода малого параметра для решения стохастических нелинейных задач теории установившейся ползучести // Вестник Чувашского государственного педагогического университета им. И.Я. Яковлева. Серия: Механика предельного состояния. 2013. № 1 (15). С. 185-194.

23. Вервейко Н.Д., Фролова О.А. Предельное осесимметричное напряжённое состояние сжимаемого сыпучего материала с цилиндрической полостью // Вестник Чувашского государственного педагогического университета им. И.Я. Яковлева. Серия: Механика предельного состояния. 2015. № 3 (25). С. 83-92.

24. Христич Д.В., Астапов Ю.В. Учёт взаимного влияния полей напряжений, деформаций и температур при решении задачи Ламе // Известия Тульского государственного университета. Естественные науки. 2015. № 1. С. 67-73.

25. Ковалев А.В., Свиридов И.Э., Щеглова Ю.Д. Применение метода возмущений при определении напряженно-деформированного состояния двухслойного слабоанизотропного стержня некругового поперечного сечения при упругопластическом кручении // Вестник Самарского государственного технического университета. Серия: Физико-математические науки. 2017. Т. 21. № 2. С. 292-307. DOI: 10.14498/vsgtu1541.

26. Чан Л.Т., Тарлаковский Д.В. Упругое моментное полупространство под действием осесимметричных нестационарных поверхностных кинематических возмущений // Проблемы прочности и пластичности. 2019. Т. 81. № 1. С. 40-52. DOI: 10.32326/1814-91462019-81-1-40-52.

27. Nilson Tadeu Mascia, Francisco Antônio Rocco Lahr. Remarks on orthotropic elastic models applied to wood // Materials Research. 2006. V. 9(3). P. 301-310. DOI 10.1590/S151614392006000300010.

28. Xiaowen Lei, Toshiaki Natsuki, Jinxing Shi, Qing-Qing Ni. Analysis of carbon nanotubes on the mechanical properties at atomic scale // Journal of Nanomaterials. 2011. V. 2011 DOI: $10.1155 / 2011 / 805313$.

29. Бакушев С.В. Дифференциальные уравнения равновесия сплошной среды для плоской деформации в декартовых координатах при билинейной аппроксимации замыкающих уравнений (геометрически линейная модель) // Региональная архитектура и строительство. 2019. № 1(38). С. 76-85.

30. Бакушев С.В. Дифференциальные уравнения равновесия сплошной среды для плоской деформации в декартовых координатах при билинейной аппроксимации замыкающих уравнений (геометрически нелинейная модель) // Региональная архитектура и строительство. 2019. № 2(39). С. 86-100.

31. Бакушев C.B. Дифференциальные уравнения равновесия осесимметричной деформации при билинейной аппроксимации замыкающих уравнений // Строительная механика и расчёт сооружений. 2019. № 1. С. 8-17.

32. Бакушев С.В. Дифференциальные уравнения равновесия центрально-симметричной деформации при билинейной аппроксимации замыкающих уравнений // Известия вузов. Строительство. 2018. № 11(719). С. 5-19.

33. Бакушев С.В. Аппроксимация диаграмм деформирования билинейными функциями // Строительная механика и расчёт сооружений. 2019. № 2 (283). С. 2-11. 
Bakushev S.V. (2021) DIFFERENTIAL EQUATIONS OF CONTINUUM EQUILIBRIUM FOR A PLANE STRAIN IN CYLINDRICAL COORDINATES AT BILINEAR APPROXIMATION OF CLOSING EQUATIONS. Vestnik Tomskogo gosudarstvennogo universiteta. Matematika $i$ mekhanika [Tomsk State University Journal of Mathematics and Mechanics]. pp. 69-85

\section{DOI $10.17223 / 19988621 / 69 / 6$}

Keywords: solid body, plane strain, cylindrical coordinates, differential equations of equilibrium, closing bilinear equations, geometrically linear model.

Problems of the formulation of differential equations of equilibrium in terms of displacements for a plane strain of continuous media at bilinear approximation of closing equations are considered leaving out of account geometric nonlinearity in the cylindrical coordinate system. Based on the assumption that the curves of volumetric and shear strain are independent from each other, six main cases of physical dependencies are considered, which are the functions of the relative position of break points on the bilinear curves of the volumetric and shear strain. Obtaining of bilinear physical dependencies is based on the calculation of secant moduli of the volumetric and shear strain. On the first line of the curves, secant moduli are constant for both volumetric and shear strain, while on the second line, the secant modulus of the volumetric strain is a function of the volumetric strain, and the secant modulus of the shear strain is a function of the shear strain intensity. Putting the corresponding bilinear physical equations into differential equations of continuum equilibrium, which disregard geometrical nonlinearity, the resulting differential equations of equilibrium are obtained in terms of displacements for a one-dimensional plane strain of continuum in the cylindrical coordinate system. These equations can be used when determining stress-strain state of continuous media under one-dimensional plane strains with no regard for geometrical nonlinearity, and whose physical relations are approximated by bilinear functions.

Sergey V. BAKUSHEV (Doctor of Technical Sciences, Professor, Penza State University of Architecture and Construction, Penza, Russian Federation). E-mail: bakuchsv@mail.ru

\section{REFERENCES}

1. Novozhilov V.V. (1958) Teoriya uprugosti [Theory of elasticity]. Leningrad: Sudpromgiz.

2. Bakushev S.V. (2013) Geometricheski i fizicheski nelineynaya mekhanika sploshnoy sredy: Ploskaya zadacha [Geometrically and physically non-linear mechanics of continuous medium: Plane problem]. Moscow: Knizhnyy dom «Librokom».

3. Rudykh O.L. (2010) Prakticheskie voprosy approksimatsii eksperimental'nykh krivykh stepennymi i drobno-lineynymi funktsiyami [Practical issues on the approximation of experimental curves by power and linear-fractional functions]. Vestnik Tomskogo gosudarstvennogo arkhitekturno-stroitel'nogo universiteta - Journal of Construction and Architecture. 1(26). pp. 110-122.

4. Gonik E.G., Kibets A.I., Petrov M.V., Fedorova T.G., Frolova I.A. (2017) Vliyanie approksimatsii diagrammy deformirovaniya na kriticheskie nagruzki pri poperechnom izgibe tsilindricheskoy obolochki [The effect of approximating deformation curves on critical load values of lateral bending of a cylindrical shell]. Problemy prochnosti i plastichnosti - Problems of Strength and Plasticity. 79(2). pp. 169-181.

5. Korol' E.Z. (2005) Opredelenie diagramm nelineynogo deformirovaniya raznosoprotivlyayushchikhsya materialov pri neodnorodnom napryazheonno-deformirovannom sostoyanii [Determination of nonlinear strain curves for materials with different resistance in an inhomogeneous stress-strain state]. Deponirovannaya rukopis' No. 86-V2005 21.01.2005.

6. Zubchaninov V.G. (2018) The general mathematical theory of plasticity and the Il'yushin postulates of macroscopic definability and isotropy. Moscow University Mechanics Bulletin. 73. pp. 101-116. DOI: 10.3103/S0027133018050011. 
7. Sadovskiy V.M. (2003) Reologicheskie modeli raznomodul'nykh i sypuchikh sred [Rheological models of hetero-modular and granular media]. Dal'nevostochnyy matematicheskiy zhurnal-Far Eastern Mathematical Journal. 4(2). pp. 252-263.

8. Morshchinina A.A. (2009) Nelineynaya osesimmetrichnaya zadacha teorii uprugosti dlya poloy sfery [Non-linear axisymmetric problem of the theory of elasticity for a hollow sphere]. Vestnik Sankt-Peterburgskogo universiteta. Matematika. Mekhanika. Astronomiya - Vestnik of Saint Petersburg University. Mathematics. Mechanics. Astronomy. 4. pp. 84-88.

9. Fedotova I.A. (2004) Matematicheskaya model' raboty nelineynogo sterzhnevogo elementa konstruktsii [A mathematical model of the operation of non-linear structural mainstay]. Izvestiya Peterburgskogo universiteta putey soobshcheniya - Proceedings of Petersburg Transport University. 1. pp. 7-12.

10. Mitskevich S.A., Krys'ko A.V., Zhigalov M.V., Krys'ko V.A. (2017) Dinamicheskaya ustoychivost' pologikh obolochek na pryamougol'nom plane s uchetom geometricheskoy i fizicheskoy nelineynosti [Dynamic stability of shallow shells on the rectangular plan, taking into account geometric and physical nonlinearity]. Problemy prochnosti $i$ plastichnosti Problems of Strength and Plasticity. 79(3). pp. 249-258. DOI: 10.32326/1814-9146-2017-793-249-258.

11. Makhmetova N.M. (2014) Ob odnom algoritme nelineynykh zadach statiki podzemnykh sooruzheniy [On the algorithm for non-linear static problems of subsurface structures]. Vestnik Kazakhskoy akademii transporta i kommunikatsiy im. M. Tynyshpaeva - The Bulletin of KazATC. 5(90). pp. 80-87.

12. Krys'ko V.A., Papkova I.V., Saltykova O.A., Babenkova T.V., Kashubina A.A. (2014) Slozhnye kolebaniya balok Eylera-Bernulli s uchetom geometricheskoy i fizicheskoy nelineynostey [Complex oscillation of the Euler-Bernoulli beams with regard to geometrical and physical nonlinearity]. Mezhdunarodnyy nauchno-issledovatel'skiy zhurnal - International Research Journal. 3-1(22). pp. 14-16.

13. Petrov V.V. (2016) Raschet neodnorodnykh po tolshchine obolochek $s$ uchetom fizicheskoy $i$ geometricheskoy nelineynostey [Calculation of inhomogeneous thickness of shells with considering physical and geometrical nonlinearities]. Academia. Arkhitektura i stroitel'stvo Academia. Architecture and Construction. 1. pp. 112-117.

14. Egorov A.V. (2018) Deformirovanie tsentral'no-szhatogo gibkogo sterzhnya [Straining an axially loaded slender column]. Inzhenernyy zhurnal: nauka i innovatsii - Engineering Journal: Science and Innovation. 4(76). pp. 1. DOI: 10.18698/2308-6033-2018-4-1750.

15. Lur'e A.I. (1980) Nelineynaya teoriya uprugosti [Nonlinear theory of elasticity]. Moscow: Nauka.

16. Demidov S.P. (1979) Teoriya uprugosti: Uchebnik dlya vuzov [Theory of elasticity: Textbook for higher education]. Moscow: Vysshaya shkola.

17. Papkovich P.F. (1939) Teoriya uprugosti [Theory of elasticity]. Moscow; Leningrad: Oborongiz.

18. Zaripov R.M., Masalimov R.B., Lisin Yu.V. (2015) Modelirovanie napryazhennodeformirovannogo sostoyaniya pryamolineynykh i krivolineynykh uchastkov truboprovoda [Modeling stress-strain state of rectilinear and curvilinear sections of the pipeline]. Neftegazovoe delo - Petroleum Engineering. 13(3). pp. 103-109.

19. Strel'nikov V.N., Sukov G.S., Voloshin A.I., Chibisov Yu.V., Lesnyak G.A. (2010) Uravneniya uprugosti $\mathrm{v}$ bipolyarnykh koordinatakh [Elastic equations in terms of bipolar coordinates]. Progressivnye tekhnologii i sistemy mashinostroeniya - Progressive Technologies and Systems of Mechanical Engineering. 2(40). pp. 248-253.

20. Buntov A.E., Gotsev D.V. (2018) Neodnorodnoe napryazhenno-deformirovannoe sostoyanie uprugogo tsilindricheskogo tela s uchetom vnutrenney struktury materiala [Inhomogeneous stress-deformed state of an elastic cylindrical body taking into account its material internal structure]. Vestnik Dagestanskogo gosudarstvennogo tekhnicheskogo universiteta. Tekhnicheskie nauki - Herald of Dagestan State Technical University. Technical Sciences. 45(1). pp. 8-21. 
21. Nemirovskiy Yu.V., Matveev K.A., Mokhovnev D.V. (2014) Ratsional'noe proektirovanie armirovannykh kol'tsevykh plastin [Efficient design of reinforced annular plates]. Problemy optimal'nogo proektirovaniya sooruzheniy: sbornik dokladov 3-y Vserossiyskoy konferentsii. pp. 272-282.

22. Radchenko V.P., Popov N.N. (2013) Ispol'zovanie metoda malogo parametra dlya resheniya stokhasticheskikh nelineynykh zadach teorii ustanovivsheysya polzuchesti [A smallparameter method applied for solving of stochastic non-linear problems of steady-state creep theory]. Vestnik Chuvashskogo gosudarstvennogo pedagogicheskogo universiteta im. I.Ya. Yakovleva. Seriya: Mekhanika predel'nogo sostoyaniya - Bulletin of the Yakovlev Chuvash State Pedagogical University. Series: Mechanics of Limit State. 1(15). pp. 185-194.

23. Verveyko N.D., Frolova O.A. (2015) Predel'noe osesimmetrichnoe napryazhennoe sostoyanie szhimaemogo sypuchego materiala s tsilindricheskoy polost'yu [ Limit axisymmetric stressful state of compressible loose material with cylindrical air space]. Vestnik Chuvashskogo gosudarstvennogo pedagogicheskogo universiteta im. I.Ya. Yakovleva. Seriya: Mekhanika predel'nogo sostoyaniya - Bulletin of the Yakovlev Chuvash State Pedagogical University. Series: Mechanics of Limit State. 3(25). pp. 83-92.

24. Hristich D.V., Astapov Yu.V. (2015) Uchet vzaimnogo vliyaniya poley napryazheniy, deformatsiy i temperatur pri reshenii zadachi Lame [Account of mutual influence of stress, strain and temperature fields at Lame problem solving]. Izvestiya Tul'skogo gosudarstvennogo universiteta. Estestvennye nauki - Izvestiya Tula State University. Natural Sciences. 1. pp. 67-73.

25. Kovalev A.V., Sviridov I.E., Shcheglova Yu.D. (2017) Primenenie metoda vozmushcheniy pri opredelenii napryazhenno-deformirovannogo sostoyaniya dvukhsloynogo slaboanizotropnogo sterzhnya nekrugovogo poperechnogo secheniya pri uprugoplasticheskom kruchenii [Application of the perturbation method for the determination of stress-strain state of a thickstyle two-layer anisotropic shaft of non-circular cross section with elastoplastic torsion]. Vestnik Samarskogo gosudarstvennogo tekhnicheskogo universiteta. Seriya: Fizikomatematicheskie nauki - Journal of Samara State Technical University. Series: Physical and Mathematical Sciences. 21(2). pp. 292-307. DOI: 10.14498/vsgtu1541.

26. Chan L.T., Tarlakovskiy D.V. (2019) Uprugoe momentnoe poluprostranstvo pod deystviem osesimmetrichnykh ne-statsionarnykh poverkhnostnykh kinematicheskikh vozmushcheniy [Elastic moment half-space under the action of axisymmetric non-stationary surface kinematic perturbations]. Problemy prochnosti i plastichnosti - Problems of Strength and Plasticity. 81(1). pp. 40-52. DOI: 10.32326/1814-9146-2019-81-1-40-52.

27. Nilson Tadeu Mascia, Francisco Antônio Rocco Lahr (2006) Remarks on orthotropic elastic models applied to wood. Materials Research. 9(3). pp. 301-310. DOI: 10.1590/S151614392006000300010.

28. Xiaowen Lei, Toshiaki Natsuki, Jinxing Shi, Qing-Qing Ni (2011) Analysis of carbon nanotubes on the mechanical properties at atomic scale. Journal of Nanomaterials. DOI: $10.1155 / 2011 / 805313$.

29. Bakushev S.V. (2019) Differentsial'nye uravneniya ravnovesiya sploshnoy sredy dlya ploskoy deformatsii $\mathrm{v}$ dekartovykh koordinatakh pri bilineynoy approksimatsii zamykayushchikh uravneniy (geometricheski lineynaya model') [Differential equations of solid environment equilibrium for flat deformation in Cartesian coordinates at bilinear approximation of trailing equations (geometrically linear model)]. Regional'naya arkhitektura i stroitel'stvo - Regional Architecture and Engineering. 1(38). pp. 76-85.

30. Bakushev S.V. (2019) Differentsial'nye uravneniya ravnovesiya sploshnoy sredy dlya ploskoy deformatsii $\mathrm{v}$ dekartovykh koordinatakh pri bilineynoy approksimatsii zamykayushchikh uravneniy (geometricheski neliney-naya model') [Differential equations of solid environment equilibrium for flat deformation in Cartesian coordinates at bilinear approximation of trailing equations (geometrically nonlinear model)]. Regional'naya arkhitektura $i$ stroitel'stvo - Regional Architecture and Engineering. 2(39). pp. 86-100.

31. Bakushev S.V. (2019) Differentsial'nye uravneniya ravnovesiya osesimmetrichnoy deformatsii pri bilineynoy approksimatsii zamykayushchikh uravneniy [Differential equations of equi- 
librium an the axially symmetric deformation at bilinear approximation trailing equations]. Stroitel'naya mekhanika i raschet sooruzheniy - Structural Mechanics and Analysis of Constructions. 1. pp. 8-17.

32. Bakushev S.V. (2018) Differentsial'nye uravneniya ravnovesiya tsentral'no-simmetrichnoy deformatsii pri bi-lineynoy approksimatsii zamykayushchikh uravneniy [Differential equations of equilibrium of central-symmetric deformation at bilinear approximation trailing equations]. Izvestiya VUZov. Stroitel'stvo - News of Higher Educational Institutions. Construction. 11(719). pp. 5-19.

33. Bakushev S.V. (2019) Approksimatsiya diagramm deformirovaniya bilineynymi funktsiyami [Approximations of warp diagrams using bilinear functions]. Stroitel'naya mekhanika $i$ raschet sooruzheniy - Structural Mechanics and Analysis of Constructions. 2(283). pp. 2-11.

Received: June 20, 2019 